\title{
Estructura poblacional, abundancia, distribución y uso de hábitat de Caiman crocodilus fuscus (Cope, 1868) en la Vía Parque Isla de Salamanca, Caribe colombiano
}

\author{
Population structure, abundance, distribution and habitat use of Caiman crocodilus fuscus \\ (Cope, 1868) in the Via Parque Isla de Salamanca, Colombian Caribbean \\ Sergio A. Balaguera-Reina ${ }^{1}$ y José F. González-Maya ${ }^{1}$ \\ ${ }^{1}$ Proyecto de Conservación de Aguas y Tierras, ProCAT Colombia, Calle 127B \# 45-76 Bogotá, Colombia \\ jfgonzalezmaya@gmail.com
}

\begin{abstract}
We analyzed the population structure and distribution of Caiman crocodilus fuscus in the Vía Parque Isla de Salamanca (VIPIS), which is considered an important protected area for this species in the country. A total of 82 individuals was registered in 12 lagoons and three creeks, covering a total of 119,02 km for each monthly sampling, with an observation mean of 21,50 \pm 7,12 individuals per survey. Mean density for lagoons was 0,063 $\pm 0,057$ ind. ha ${ }^{-1}$ and 1,37 $\pm 1,218$ ind. $\mathrm{km}^{-1}$ for creeks. Population structure distribution was: $36.59 \%$ for class I, $53.66 \%$ for class II, $8.54 \%$ for class III and $1.22 \%$ for class IV. There was no relationship between presence of Caiman c. fuscus and vegetation type $(P=0.64)$. Results indicate that the population of Caiman $c$. fuscus is distributed along the entire study area; however, it is possible that they are still subject of extraction and exploitation by nearby communities. Efficient exploitation controls, population monitoring, vigilance and protected areas adaptive management could be the best option for the long-term conservation of the species.
\end{abstract}

Key words: Caiman, density, population structure, national parks

\section{Introducción}

De las 23 especies de cocodrilianos descritas a nivel global, diez se encuentran en América del Sur y seis tienen distribución en Colombia (King \& Burke 1989, Barahona et al. 1996, Ross 1998). Esta riqueza ha despertado a través del tiempo un alto interés en las poblaciones colombianas, mostrándose su relevancia a nivel cultural e industrial durante el siglo XX dentro de las festividades locales caribeñas y el aprovechamiento marroquinero (Chiriví 1973, Medem 1981, Ulloa-Delgado \& SierraDíaz 2002). Esto generó que para la segunda mitad del siglo pasado, la explotación irracional de este recurso biológico y el deterioro de su hábitat, generara la reducción y desaparición local de algunas poblaciones de especies explotadas comercialmente como Crocodylus intermedius (Graves 1819), C. acutus (Cuvier 1807),
Resumen.- Se analizó la estructura poblacional y distribución de Caiman crocodilus fuscus en la Vía Parque Isla de Salamanca (VIPIS), considerada un área importante para esta especie en el país. Se registró un total de 82 individuos en 12 ciénagas y tres caños, recorriendo $119,02 \mathrm{~km}$ por muestreo mensual, con un promedio de observación de 21,50 \pm 7,12 individuos por muestreo. La densidad promedio fue de $0,063 \pm 0,057$ ind. ha ${ }^{-1}$ para ciénagas y de 1,37 $\pm 1,218$ ind. $\mathrm{km}^{-1}$ para caños. La estructura poblacional estuvo distribuída en 36,59\% para la clase I, 53,66\% para la clase II, 8,54\% para la clase III y 1,22\% para la clase IV. No se encontró relación entre la presencia de Caiman c. fuscus y las cuatro categorías de hábitat sugeridas en el presente estudio $(P=0,64)$. Los datos indican que la población de Caiman c. fuscus se encuentra distribuída a lo largo de toda el área de estudio, posiblemente sujetas aún a extracción y explotación por parte de las comunidades aledañas. Controles eficientes de la explotación, monitoreos continuos sobre la especie y manejo adaptativo de las áreas protegidas, pueden ser las mejores opciones para la conservación de esta especie a largo plazo.

Palabras clave: Caimán, densidad, babilla, estructura poblacional, parques nacionales

Caiman crocodilus (Linnaeus 1758) y Melanosuchus niger (Spix 1825) (Rodriguez 2000, Ulloa-Delgado \& Sierra-Díaz 2002).

Caiman crocodilus fuscus, conocido comúnmente como babilla en Colombia, no ha sido ajeno a esta presión, debido principalmente a la sobre-explotación en su hábitat natural, alcanzando valores aproximados de hasta 400.000 pieles anuales entre inicios de 1950 y 1980 (MacGregor 2006). Debido a las causas anteriormente mencionadas, C. c. fuscus fue catalogado en el Apéndice II de CITES. En el caso específico de Colombia, según el censo realizado por el Ministerio del Medio Ambiente entre los años de 1994 y 1996 (Barahona et al. 1996), se consideró como 'caso inesperado' al encontrarse un número reducido de individuos y poblaciones fragmentadas (Rodríguez 2002). Para el área 
correspondiente a la Vía Parque Isla de Salamanca aún no se cuenta con conteos y estudios poblacionales sistemáticos, a pesar de ser considerada como un área de importancia para esta especie, por lo que el presente estudio representa un importante acercamiento a la situación de C. c. fuscus en la región.

El objetivo de este estudio fue evaluar la población de Caiman c. fuscus, teniendo en cuenta su distribución, abundancia, densidad, estructura poblacional y uso de hábitat en la VIPIS, con el fin de avanzar en el conocimiento en áreas relevantes para la protección y conservación de las poblaciones silvestres de cocodrilianos en Colombia.

\section{Material y métodos}

\section{Área de estudio}

La Vía Parque Isla de Salamanca (VIPIS) se ubica en el departamento de Magdalena, en jurisdicción de los municipios de Pueblo Viejo y Sitio Nuevo del mismo

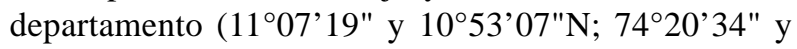
$\left.74^{\circ} 51^{\prime} 00^{\prime \prime} \mathrm{W}\right)$, limitando al norte con el mar Caribe, al sur con el complejo cenagoso Pajarales, caño Clarín Nuevo y el municipio de Sitio Nuevo, al este con el municipio de Pueblo Viejo y al oeste con el río Magdalena, Colombia (Fig. 1). Cuenta con una extensión de 56.200 ha de las cuales el 50\% son áreas continentales (UAESPNN 2004). Además se encuentra en la región

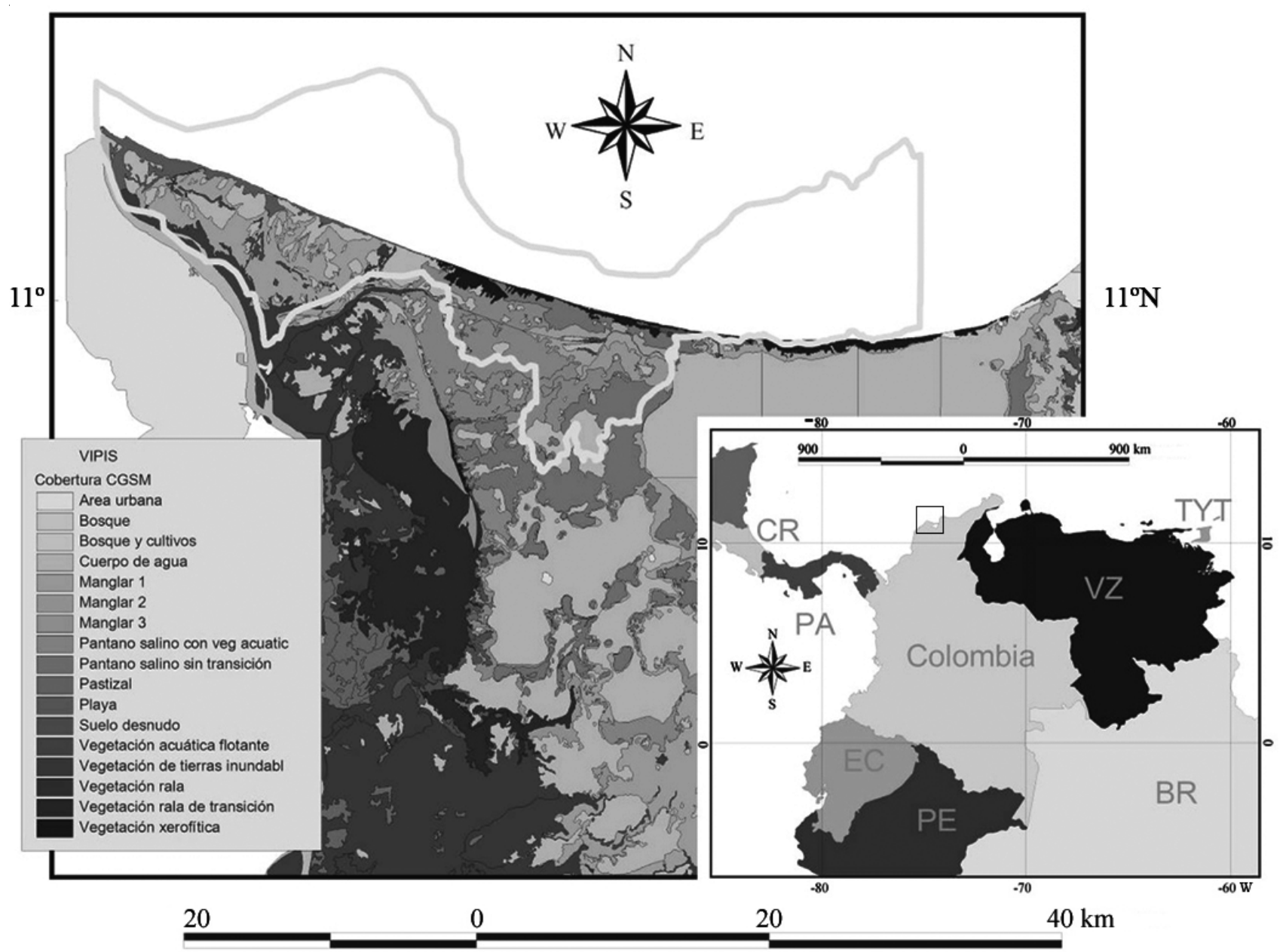

Figura 1

Ubicación del área de estudio, Caribe colombiano

Study area, Colombian Caribbean 
septentrional de la eco-región Ciénaga Grande de Santa Marta que constituye el complejo lagunar costero más grande de Colombia $\left(4.280 \mathrm{~km}^{2}\right.$ ) y está incluida dentro de la Convención RAMSAR y reconocida como Reserva de la Biosfera por la UNESCO (Moreno-Bejarano \& Álvarez-León 2003). El área de la reserva corresponde a la zona de vida denominada Bosque Seco Tropical y una franja angosta corresponde a Bosque Muy Seco Tropical (Tavera \& Gamba 2001). El clima se caracteriza por tener una temperatura promedio anual de $28^{\circ} \mathrm{C}$, una precipitación que oscila entre 400,1 y 760,2 mm año ${ }^{-1}$, una humedad relativa media anual de $75 \%$ y un potencial de evapotranspiración total anual de $1.707 \mathrm{~mm}$, implicando un déficit hídrico todo el año (UAESPNN 2004).

\section{Censos nocturnos}

Con base en los Sistemas de Información Geográfica de la Vía Parque, se subdividió el área en cinco rutas, las cuales fueron evaluadas con un esfuerzo muestreal de 5 días mensuales entre septiembre y diciembre de 2006 (época final de lluvias y comienzo de la época seca), abarcando de sur a norte y de occidente hasta la parte media de la VIPIS. Los días de muestreo se escogieron bajo el criterio de las fases lunares (luna nueva). Se determinó a las ciénagas y los caños ubicadas dentro de estas rutas como unidades muestreales independientes basados en los planteamientos de Sánchez-Páez et al. (2004). Se realizaron recorridos nocturnos diarios por cada ruta a lo largo de los cinco días mensuales en una lancha con motor fuera de borda (40 Hp) entre las 19:00 y las 02:00 h a una velocidad máxima de $7 \mathrm{~km} \mathrm{~h}^{-1}$. Se utilizaron lámparas frontales de corto alcance y halógenas de 2.000.000 candelas de potencia, usando como fuente de energía una batería de automotor Mac 550 AHm de 12 V (Ulloa-Delgado \& Sierra-Díaz 2002).

Los animales fueron detectados por medio de la luz reflejada por sus ojos (tapetum lucidum). Al ser avistado el animal se procedía a acercarse al máximo para así determinar con mayor veracidad el tamaño del individuo (Chabreck 1966, Medem 1981, Salas 1985, INDERENA 1994). Los individuos se agruparon según la clasificación de Ayarzagüena (1983), que segrega en cuatro clases de tamaño (Clase I: $\leq 50 \mathrm{~cm}$, Clase II: 50,1-120 cm, Clase III: $120,1-180 \mathrm{~cm}$, Clase IV: $\geq 180,1 \mathrm{~cm})$, las cuales a su vez conservan ciertas características biológicas (Velasco \& Ayarzagüena 1995).

Se realizó una descripción del tipo de vegetación y su estructura en los sitios donde se encontraban los individuos avistados. Se utilizó una tabla de contingencia (estadístico Chi-cuadrado) para determinar si existía relación entre la presencia o ausencia de $C$. $c$. fuscus y las estructuras de hábitat presentes en el área (vegetación dominada por Rhizophora mangle; área dominada por neumatóforos de Avicennia germinans; vegetación dominada por especies flotantes y espejo de agua) y una regresión lineal para determinar si existe una relación directa entre el área del cuerpo de agua y la abundancia. A nivel espacial se generó una aproximación geográfica a la distribución de la especie con base en los avistamientos realizados a lo largo del estudio, con el fin de tener una perspectiva de la extensión del hábitat ocupado por la especie y poder entender la magnitud y distribución de la especie y las amenazas potenciales.

\section{Resultados}

Se recorrieron $119,02 \mathrm{~km}$ por muestreo mensual abarcando desde el costado occidental de la Vía Parque hasta su parte media, con un área para ciénagas de 2.394 ha de espejo de agua y una distancia para caños de 23,62 $\mathrm{km}$. Se visitaron 14 ciénagas y 10 caños (UAESPNN 2004), correspondiendo al 48,27\% de las ciénagas y el 76,92\% de los caños existentes en la VIPIS. La especie presentó una amplia distribución a lo largo de la Vía Parque, desde el costado más oriental muestreado (ciénaga Cuatro Bocas) hasta el límite occidental de la VIPIS (río Magdalena), siendo avistada en el 78,57\% de las ciénagas muestreadas. Se observó un mayor número de individuos en el costado occidental de la Vía Parque disminuyendo la cantidad de avistamientos hacia el centro del mismo, aumentando nuevamente en la ciénaga Manatíes y hacia el sur en el caño Clarín Nuevo, en donde se realizaron observaciones a lo largo de todo el trayecto muestreado.

Se realizaron 82 avistamientos de C. c. fuscus en toda el área a lo largo de los cuatro meses de estudio (promedio $21,50 \pm 7,12$ ) en 12 ciénagas y tres caños, obteniendo un mayor número de observaciones en las ciénagas en relación a los caños en proporción 3,82:1, respectivamente. Se observó una variación significativa de la densidad estimada entre ciénagas, obteniéndose un valor promedio de 0,063 $\pm 0,057$ ind. $\mathrm{ha}^{-1}$ al igual que en la abundancia estimada entre caños, mostrándose un valor promedio de 1,01 \pm 1,218 ind. $\mathrm{km}^{-1}$ (Tabla 1). La distribución de clases por caños y ciénagas estuvo caracterizada por la presencia de las cuatro clases de tamaño, con un mayor número de juveniles (clase I $36,59 \%$ y II 53,66\%) seguido por sub-adultos (clase III $8,54 \%)$. La clase IV contó con un único registro en caños y ninguno en ciénagas (1,22\%) (Fig. 2). En cuanto a la distribución de las clases, se registró un claro dominio de la clase II en la mayoría de las ciénagas y caños recorridos, con excepción de la ciénaga Marchena. En el caso de los individuos sub-adultos (clase III) se observó una alta 
Tabla 1

Estructura poblacional y distribución de Caiman crocodilus fuscus por sitio y ruta de muestreo

Population structure and distribution of Caiman crocodilus fuscus according to site and sampling route

\begin{tabular}{|c|c|c|c|c|c|c|c|}
\hline Ruta & Caños/Ciénagas & $\begin{array}{l}\text { Perímetro } \\
(\mathrm{km})\end{array}$ & Área (ha) & $\begin{array}{c}\mathrm{N}^{\circ} \\
\text { individuos }\end{array}$ & $\begin{array}{c}\text { Clase } \\
\text { Tamaño }\end{array}$ & $\begin{array}{l}\text { Densidad } \\
\left(\text { Ind. } \mathrm{ha}^{-1} \text { ) }\right.\end{array}$ & $\begin{array}{l}\text { Abundancia } \\
\text { (Ind. } \mathrm{km}^{-1} \text { ) }\end{array}$ \\
\hline \multirow[t]{9}{*}{1} & Cié. Dársena & 8,80 & 143,12 & 10 & I, II, III & 0,0699 & - \\
\hline & Cié. Poza Verde & 9,20 & 333,89 & 11 & I, II, III & 0,0329 & - \\
\hline & Cié. Loro & 5,10 & 45,10 & 4 & I, II & 0,0887 & - \\
\hline & Cié. Torno & 9,90 & 383,77 & 4 & I, II, III & 0,0104 & - \\
\hline & Cié. Baco & 3,10 & 26,03 & 4 & I, II & 0,1536 & - \\
\hline & Caño Limón & 2,60 & 2,25 & 1 & III & & 0,38 \\
\hline & Caño Loro & 0,70 & 2,28 & - & - & - & - \\
\hline & Caño Almendros & 0,30 & 0,15 & - & - & - & - \\
\hline & Total & 39,70 & 936,59 & 34 & - & - & - \\
\hline \multirow[t]{7}{*}{2} & Cié. Playa del Perro & 5,90 & 59,15 & 10 & I, II & 0,1691 & - \\
\hline & Cié. Lirial & 12,80 & 190,64 & - & - & - & - \\
\hline & Cié. Las Moras & 6,60 & 63,22 & 3 & I, II & 0,0475 & - \\
\hline & Cié. Marchena & 9,30 & 136,98 & 2 & I, III & 0,0146 & - \\
\hline & Cié. Buchá & 1,50 & 6,80 & - & - & - & - \\
\hline & Suiches de Marchena & 1,70 & 0,34 & - & - & - & - \\
\hline & Total & 37,80 & 457,13 & 15 & - & - & - \\
\hline \multirow[t]{6}{*}{3} & Cié. del Medio & 3,40 & 67,85 & 1 & II & 0,0147 & - \\
\hline & Cié. Atascosa & 7,00 & 166,06 & - & - & - & - \\
\hline & Caño el Dedo & 1,10 & 0,11 & 3 & II & - & 2,73 \\
\hline & Caño Las Lanchas & 1,10 & 0,74 & - & - & - & - \\
\hline & Caño Clarín Viejo & 0,90 & 2,15 & - & - & - & - \\
\hline & Total & 13,50 & 236,91 & 4 & - & - & - \\
\hline \multirow[t]{7}{*}{4} & Cié. Cuatro Bocas & 8,80 & 578,91 & 2 & I, II & 0,0035 & - \\
\hline & Cié. Manatíes & 4,00 & 164,46 & 14 & I, II & 0,0851 & - \\
\hline & Caño Palmitas & 1,00 & 3,93 & - & - & - & - \\
\hline & Caño Manatíes & 0,42 & 0,06 & - & - & - & - \\
\hline & Caño Clarín Nuevo & 13,00 & 15,45 & 13 & I, II, III, IV & - & 1,00 \\
\hline & Total & 27,22 & 762,81 & 29 & - & - & - \\
\hline & Total general & 119,02 & 2394,44 & 82 & & & \\
\hline
\end{tabular}

disgregación de estos dentro de la Vía Parque, registrándose solo en la ciénaga Poza Verde dos subadultos, al igual que en caño Clarín Nuevo un individuo clase III y uno clase IV. El mayor número de individuos Clase I se presentó en la parte central del área de estudio, siendo esta misma zona el lugar en donde no se presentaron avistamientos de animales adultos (clases III y IV) (Fig. 3).

Aunque la cantidad de individuos avistados en los caños fue baja en comparación con las ciénagas, los caños Clarín Nuevo y El Dedo presentaron las abundancias más altas obtenidas a lo largo de todo el estudio (1,00 y 2,73 ind. $\mathrm{km}^{-1}$ respectivamente), siendo este primero el único sitio donde fueron observados individuos de las cuatro clases de tamaño. En las ciénagas por su parte, los valores de densidad oscilaron entre 0,169 y 0,003 ind. ha-1, obtenidos en Playa del Perro y Cuatro Bocas, respectivamente, no observándose individuos de clase IV y la cantidad de $C$. $c$. fuscus clases I y II fue mucho mayor con respecto a la clase III.

A nivel de hábitat, los individuos se encontraron en mayor proporción asociados a vegetación flotante (54,12\%), seguida por estructuras de especies arbóreas (32,94\%) y sobre el espejo de agua sin cobertura vegetal alguna (12,94\%). Sin embargo, no se encontró relación entre la presencia de $C$. $c$. fuscus y las cuatro categorías de hábitat del estudio ( $\left.P=0,12, \mathrm{v}=3, X^{2}=5,69\right)$. Tampoco se encontró una regresión lineal entre la abundancia y el 


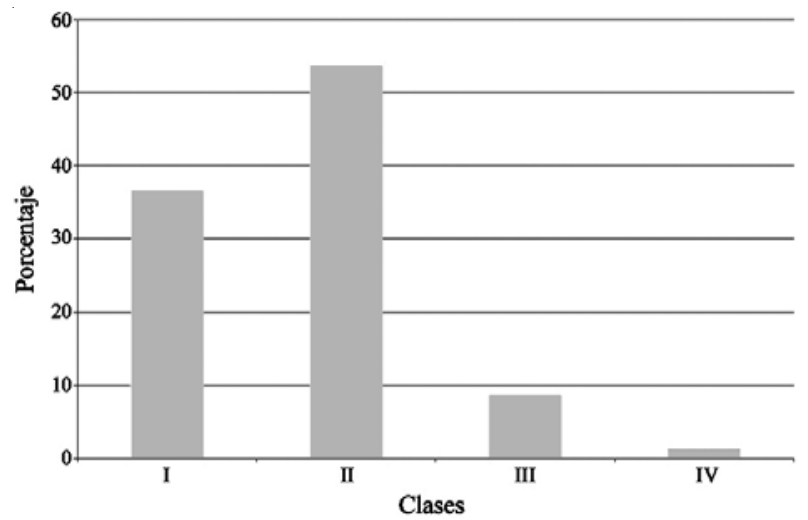

Figura 2

Distribución por clases de tamaño de Caiman crocodilus fuscus

Size class distribution of Caiman crocodilus fuscus área del cuerpo de agua $(P=0,28)$. En el caso de las variables físicas medidas, la salinidad, la temperatura del agua y la temperatura del aire presentaron valores promedio de 2,44 $\pm 3,10$ ups, $29,50 \pm 0,80{ }^{\circ} \mathrm{C}$ y 28,17 \pm $1,03{ }^{\circ} \mathrm{C}$, respectivamente, siendo bajos los valores de fluctuación.

Los sitios en donde fueron avistadas las babillas presentaron características relevantes respecto a los demás cuerpos de agua, como una alta proporción de componente vegetal tanto flotante como manglárico, además de una baja incidencia del viento debido a la cobertura boscosa existente. A nivel de abundancia, fue más relevante la vegetación flotante $(54,12 \%)$, siendo la característica más notable en las estaciones con mayor número de avistamientos (ciénagas Manatíes, Poza Verde, Playa del Perro y Dársena).

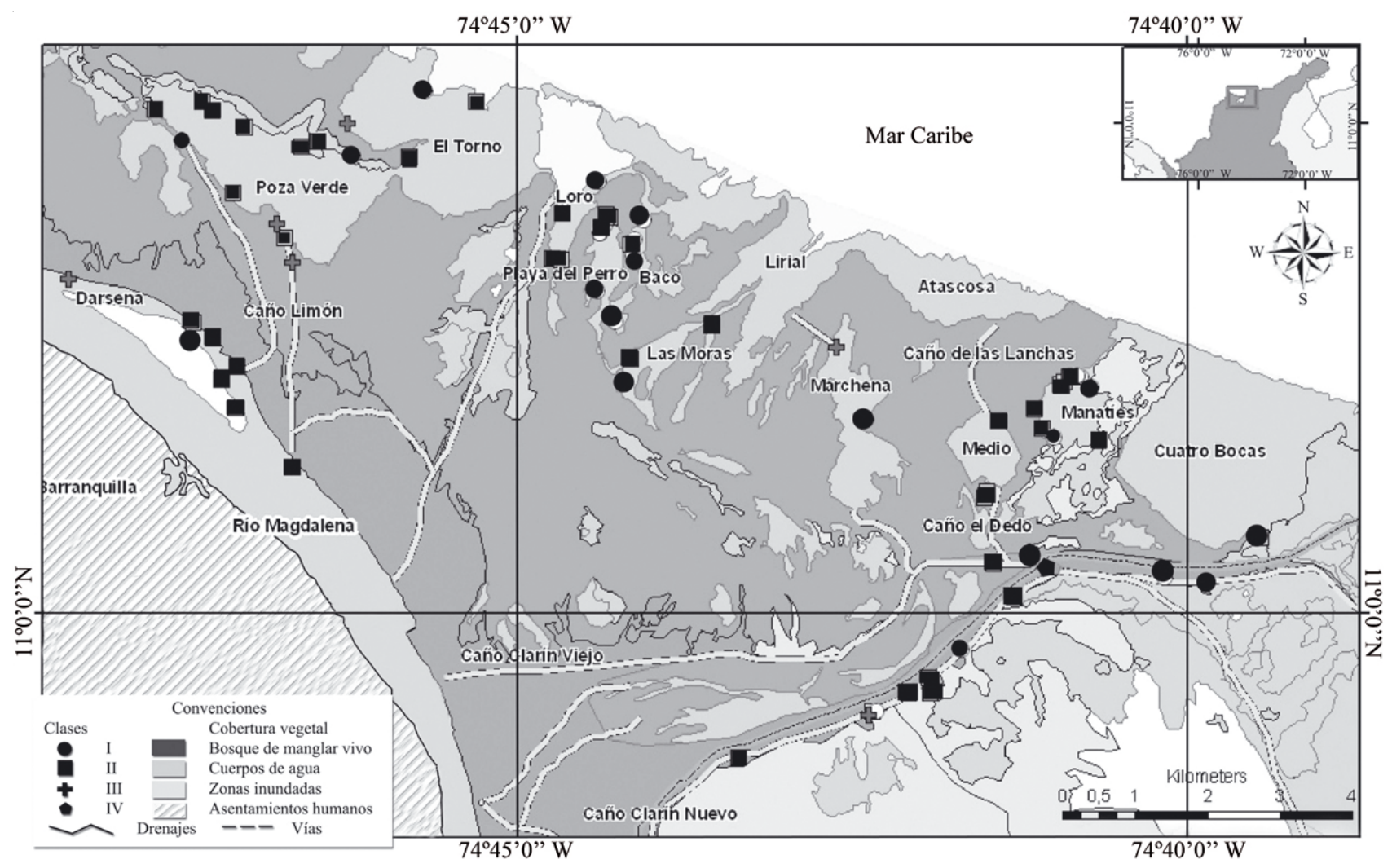

Figura 3

Distribución espacial de los individuos de Caiman c. fuscus por clases de tamaño en el VIPIS

Spatial distribution of individuals of Caiman c. fuscus per size class in the VIPIS 


\section{Discusión}

La Vía Parque Isla de Salamanca se muestra como un área de importancia como hábitat para la babilla, ya que en más de la mitad de los sitios muestreados (60\%) fue registrada la especie. Se observó una mayor preferencia hacia las ciénagas que a los caños siendo una característica típica para esta especie, lo cual coincide con lo registrado por Ulloa-Delgado \& Cavanzo-Ulloa (2003) en áreas cercanas (bahía de Cispatá, departamento de Córdoba). Esta especie se encontró asociada en mayor proporción a áreas con cobertura vegetal flotante, las cuales les proporcionan refugio tanto de posibles depredadores como para pasar inadvertidos para las presas, siendo esto registrado en estudios de hábitat de esta especie tanto a nivel nacional (Chiriví 1973, Medem 1981, Barahona et al. 1996, Rodriguez 2000, Ulloa-Delgado \& CavanzoUlloa 2003, Forero-Medina et al. 2006) como internacional (Ayarsagüena 1983, Cerrato 1991, Velasco \& Ayarsagüena 1995, De la Ossa 1996). Se considera que las poblaciones sujetas a extracción permanente presentan cierta tendencia a ubicarse en mayor proporción en los sitios más alejados y escondidos para el hombre siendo difícil su observación (Medem 1981, UlloaDelgado \& Cavanzo-Ulloa 2003), reduciéndose la presencia de individuos principalmente adultos en zonas con características óptimas para su desarrollo, pero con influjo humano (Medem 1981, Ulloa-Delgado \& Cavanzo-Ulloa 2003).

Aunque factores como la temperatura del agua y aire limitan la distribución de las babillas dadas sus características fisiológicas; el bajo ámbito de fluctuación que se registra en las aguas del Caribe colombiano a nivel continental respecto a estas variables genera que no sean una limitante en la distribución de estos individuos (UlloaDelgado \& Cavanzo-Ulloa 2003, Balaguera-Reina 2007). Es por esto que la distribución de los individuos parece estar relacionada con dos factores principales: vegetación y viento, ya que la salinidad no presentó un amplio valor de fluctuación dentro del presente estudio y el área de los cuerpos de agua tampoco presentó una relación estadística significativa con la presencia o abundancia de estos. Aunque el viento no fue un parámetro medido dentro del estudio, al parecer la velocidad de este es un factor relevante en la distribución de los crocodílidos (Cerrato 1991). En lugares donde la cobertura vegetal era baja y existía una alta influencia de los vientos sobre los cuerpos de agua, se observó la tendencia a no avistarse babillas debido a que el movimiento continuo de las aguas impide el desarrollo de las técnicas de caza de estos individuos (Medem 1981), ya que prefieren habitar remansos, lagunas y zonas de aguas lénticas (De la Ossa 1996), mostrándose estas condiciones en ciénagas como Atascosa y El Lirial las cuales no presentaron avistamientos. Ciénagas que se encontraban bordeando la costa como Cuatro Bocas, El Torno y Poza Verde, presentaron la tendencia a registrar babillas en su parte sur en donde no existía alta dinámica pero sí una alta concentración vegetal. A su vez, la alta cantidad de registros observados en los primeros meses de estudio (época de lluvias), cuando casi la tercera parte de los animales fueron avistados, y la baja cantidad de registros realizados en diciembre (época seca), concuerdan con los cambios pluviométricos y eólicos en la zona (llegada de mayor intensidad de los vientos alisios del noreste; Bernal 2004).

La heterogeneidad entre los sitios de muestreo se explica en parte por la diferencia entre los afluentes hídricos que alimentan el área, puesto que existe un gradiente de occidente a oriente y de norte a sur de carácter halo-hídrico, dado por la influencia del río Magdalena y la entrada de aguas marinas. Este factor, sumado a eventos como el viento y la pluviosidad, generan condiciones físico-químicas que influyen en la dinámica biológica, y por ende, en la distribución de esta especie, agregándolos o disgregándolos según sea el caso, a zonas con características favorables a su desarrollo (Thorbjarnarson 1989).

Otros factores a considerar están relacionados con el contacto constante con lanchas, ruido de motores, pescadores y cazadores, lo cual genera cambios en el comportamiento, que de una u otra manera afectan la determinación poblacional, haciéndolos mas crípticos y difíciles de avistar, siendo, por otro lado, los animales que han presentado poca o nula presión de caza más susceptibles a ser contados (Ron et al. 1998).

La estructura poblacional mostró estar relacionada con fuertes alteraciones por la falta de adultos, lo que puede influenciar el crecimiento poblacional. Por lo tanto es factible suponer, que tanto a nivel de ciénagas como de caños, la población podría encontrase en desequilibrio, dada la distribución por clases que presentan, mostrando cierto parecido con lo propuesto por Ayarsagüena (1983) para poblaciones de babilla sobre-explotadas en los llanos inundables venezolanos. Al unificar el total de observaciones de $C$. c. fuscus encontrados, tanto en ciénagas como en caños, se sigue observando una estructura poblacional posiblemente afectada por la extracción de individuos adultos, siendo este un factor determinante en el mantenimiento de la población en el tiempo. Este comportamiento también fue registrado en los seguimientos realizados por el Ministerio del Medio Ambiente (1994 a 1997), en donde proponen a la babilla como un 'caso inesperado' al encontrar bajas abundancias y fragmentación de poblaciones (densidad promedio de 0 a 1,5 ind. ha ${ }^{-1}$ en todo el territorio nacional), siendo las 
cuencas del Magdalena medio, el Cauca y el alto Magdalena las áreas donde se registraron los valores de densidad más elevados (0,45 ind. ha-1) (Rodríguez 2000).

El valor de densidad promedio obtenido para el total de ciénagas $(0,063$ ind. ha-1 $\pm 0,057)$ superan los estimado por Rodríguez (2000) en el censo para C. c. fuscus realizado entre los años 1994 y 1996 para el bajo Magdalena, quien propuso una densidad media para esta especie de 0,012 ind. ha-1, lo que equivale a encontrar un ejemplar por cada 80 hectáreas de superficie acuática. La estructura poblacional registrada en el presente trabajo para las babillas concuerda con lo encontrado por Rodríguez (2000) para toda el área del Caribe, donde la estructura demográfica corresponde, de acuerdo con los criterios establecidos por Velasco \& Ayarsagüena (1995), a la típica de poblaciones explotadas. Además, otros estudios realizados en el área con los habitantes locales plantean un aprovechamiento indirecto, pesca y/o captura incidental, por parte de estos sobre las poblaciones de babilla que se encuentran tanto dentro como fuera de la jurisdicción de la Vía Parque, siendo utilizado como producto de venta y/o alimentario (Balaguera-Reina \& González-Maya en prensa); ello, sumado con la estructura demográfica registrada en el presente estudio, ratifican la alteración existente por parte del componente humano sobre las poblaciones de C. c. fuscus en el área, y como se ha inferido para otras especies similares (Crocodylus acutus) en la misma Área Protegida (Balaguera-Reina \& Gonzalez-Maya 2008).

El aprovechamiento de fauna y flora en esta área natural es algo que ha sido registrado desde hace ya bastante tiempo (Chiriví 1973, UAESPNN 2004, Castaño-Mora \& Álvarez-León 2003) ya que al encontrarse ubicada dentro de la ecoregión Ciénaga Grande de Santa Marta, la cual posee alrededor de 10 municipios dentro de su territorio (Sánchez-Páez et al. 1997), y limitar en su costado occidental con el río Magdalena y la ciudad de Barranquilla, está expuesta a una continua interacción por parte de los pobladores locales con los recursos biológicos, extrayendo de manera cotidiana fauna y flora, siendo la producción de carbón de mangle junto con el aprovechamiento pesquero uno de los flagelos que más afectan el área (UAESPNN 2004).

En términos generales, las poblaciones de C. c. fuscus en la Vía Parque Isla de Salamanca muestran una estructura poblacional sometida a presión por extracción y cacería; sin embargo, estas poblaciones se encuentran dispersas a lo largo de toda el área protegida, por lo que el control de la explotación furtiva o su regulación y manejo sostenible, pueden resultar en una buena medida de conservación para el mantenimiento a largo plazo de especie.

\section{Agradecimientos}

A la Unidad Administrativa Especial del Sistema de Parques Nacionales Naturales (UAESPNN) seccional Caribe, y en especial al personal de la Vía Parque Isla de Salamanca (VIPIS) por apoyar a nivel personal, logístico y económico la presente investigación. Al zoocriadero Reptiles de Bolívar Ltda. (REPTIBOL LTDA,) por los aportes económicos para la cofinanciación de la investigación. A Armando Escobedo, Giovanni UlloaDelgado, Arturo Acero y Adolfo Sanjuán por la asistencia técnica en todos los aspectos relacionados con el mismo. A Diego Zarrate, Amancay de Atacama Cepeda y Mauricio González por su revisión, a los dos revisores anónimos por sus sugerencias y comentarios.

\section{Literatura citada}

Ayarzagüena J. 1983. Ecología del caimán de anteojos (Caiman crocodilus L.) en los llanos de Apure (Venezuela). Doñana, Acta Vertebrata, número especial 10: 45-117.

Balaguera-Reina SA. 2007. Distribución espacial y descripción de hábitat de los Crocodylia del Parque Nacional Natural Vía Isla de Salamanca, Caribe colombiano. Tesis de Biología Marina, Universidad de Bogotá Jorge Tadeo Lozano. Bogotá, 115 pp.

Balaguera-Reina SA \& JF González-Maya. 2008. Population structure, density, and habitat of Crocodylus acutus Cuvier 1807 in the Via Parque Isla de Salamanca, Magdalena department, Colombia. Herpetotropicos 4(2): 59-63.

Balaguera-Reina SA \& JF González-Maya. En prensa. Percepciones, conocimiento y relaciones entre los Crocodylia y dos poblaciones humanas cercanas a la Vía Parque Isla de Salamanca (Caribe colombiano). Acta Biológica Panamensis 1(1).

Barahona S, P Bonilla, A Martínez, H Naranjo \& MA Rodríguez. 1996. Estado, distribución, sistemática y conservación de los Crocodylia colombianos. Censo 1994 1996, pp. 32-51. Ministerio del Medio Ambiente, Convención sobre el Comercio Internacional de Especies Amenazadas de Fauna y Flora Silvestres, CITES. Santa Fe de Bogotá.

Barrera L. 2004. Estado actual de un relicto poblacional del Caimán agujo (Crocodylus acutus Cuvier, 1807) en una zona del Magdalena Medio, 8 pp. Pro Aves Colombia, Fundación Omacha. Bogotá.

Cerrato C. 1991. Composición y tamaño de las poblaciones silvestres de caimanes (Caiman crocodilus chiapasus) y cocodrilos (Crocodylus acutus) de la costa Caribe de Honduras, Centro América. Tesis de maestría en Manejo y Conservación de Vida Silvestre. Programa Regional en Manejo de Vida Silvestre para Mesoamérica y el Caribe. Universidad Nacional, Heredia, 142 pp. 
Chabreck RH. 1966. Methods of determining the size and composition of alligator population in Louisiana. Proceedings of the 20th Annual Conference South East Association on Game and Fish Commission 20: 105-112.

Chiriví H. 1973. Contribución al conocimiento de la babilla (Caiman crocodilus) con notas acerca de su manejo y de otras especies de crocodilia neotropicales, 100 pp. INDERENA, Barranquilla.

De la Ossa-Velásquez J. 1996. Colonización y ocupación territorial de lagunas artificiales por Caiman crocodilus fuscus (Cope, 1868) Crocodylia: Alligatoridae. En: 'Crocodiles', Proceedings of the $13^{\text {th }}$ Working Meeting of the Crocodile Specialist Group. Santa Fe, Argentina. IUCNThe World Conservation Union, Gland, Switzerland, pp. 117-130.

Forero-Medina G, O Castaño-Mora \& M Rodríguez-Melo. 2006. Ecología de Caiman crocodilus fuscus en San Andrés isla, Colombia: un estudio preliminar. Caldasia 28(1): 115124.

INDERENA 1994. Manual para la evaluación de poblaciones de Crocodylia en Colombia, 120 pp. Instituto Nacional de los Recursos Naturales, Bogotá.

King FW \& RL Burke. 1989. Crocodilian, tuatara and turtle species of the world: A taxonomic and geographic reference, 216 pp. Association of Systematic Collections, Washington

MacGregor J. 2006. The call of the wild: captive Crocodilian production and the shaping of conservation incentives, 44 pp. TRAFFIC International, Cambridge.

Medem F. 1981. Los Crocodylia de Sur América, Volumen 1: Los crocodylia de Colombia, 354 pp. Colciencias, Bogotá.

Moreno-Bejarano $M$ \& $R$ Álvarez-León. 2003. Fauna asociada a los manglares y otros humedales en el DeltaEstuario del río Magdalena, Colombia. Revista de la Academia Colombiana de la Ciencia 27(105): 517-534.

Rodríguez M. 2000. Estado y distribución de los Crocodylia en Colombia, 71 pp. Instituto Alexander Humboldt, Bogotá.

Rodríguez M. 2002. Caiman crocodilus fuscus. En: CastañoMora GV (ed). Libro rojo de reptiles de Colombia. Libros rojos de especies amenazadas de Colombia, pp. 10-15. Instituto de Ciencias Naturales, Universidad Nacional de Colombia, Ministerio del Medio Ambiente, Conservación Internacional -Colombia, Bogotá.

Ron RS, A Vallejo \& E Andanza. 1998. Human influence on the wariness of Melanosuchus niger and Caiman crocodilus in Cuyabeno, Ecuador. Journal of Herpetology 32(3): 320-324.

Ross JP. 1998. Crocodiles, status survey and conservation action plan, 96 pp. IUCN/SSC Crocodiles Specialist Group, IUCN, Gland, Switzerland and Cambridge.
Salas CE. 1985. Contribución al conocimiento sobre el manejo del Crocodylus acutus Cuvier (Crocodylia, Crocodylidae) en el Refugio Nacional de Fauna Silvestre Dr. Rafael Lucas Rodríguez Caballero, 49 pp. Universidad de Costa Rica, San José.

Sánchez-Páez H, R Álvarez-León, F Pinto-Nolla, AS Sanchez Alferez, JC Pino-Renjifo, I Garcia-Hansen, \& MT Acosta-Peñaloza. 1997. Diagnóstico y zonificación preliminar de los manglares del Caribe colombiano. En: Sánchez-Páez H \& R Álvarez-León (eds). Proyecto PD 171/ 91 Rev. 2 Fase II (Etapa 2) Conservación y manejo para el uso múltiple y desarrollo de los manglares en Colombia, pp. 122-129. Ministerio del Medio Ambiente / OIMT. Santa Fe de Bogotá.

Sánchez-Páez H \& R Álvarez-León. 1997. Diagnóstico y zonificación preliminar de los manglares del Caribe de Colombia, 250 pp. Ministerio del Medio Ambiente. Organización Internacional de Maderas Tropicales. Dirección de proyectos de repoblación y ordenación forestal. Proyecto PD 171-91 Rev. 2 (F) Fase 1. Unión Gráfica, Santa Fe de Bogotá.

Sánchez-Páez H, G Ulloa-Delgado \& H Tavera-Escobar. 2004. Manejo integral de los mangles por comunidades locales Caribe de Colombia, 355 pp. Ministerio de Ambiente Vivienda y Desarrollo Territorial, Dirección de Ecosistemas, Bogotá.

Tavera H \& N Gamba. 2001. Caracterización de la vegetación de la Vía Parque Isla de Salamanca, Magdalena, Colombia. Tesis de Ingeniería Forestal, Universidad Distrital Francisco José de Caldas, Bogotá, 310 pp.

Thorbjarnarson JB. 1989. Ecology of the American crocodile, Crocodylus acutus. En: Crocodiles: Their ecology, management and conservation. A Special Publication of the Crocodile Specialist Group. IUCN, Gland, pp. 228-258.

UAESPNN 2004. Plan de manejo, Vía Parque Isla de Salamanca 2005-2009, 356 pp. Dirección Territorial Costa Atlántica, Barranquilla.

Ulloa-Delgado G \& C Sierra-Díaz. 2002. Cocodrilos y manglares de la bahía de Cispatá, departamento de Córdoba-Colombia. Fase I: Caracterización y diagnóstico de las poblaciones de Crocodylus acutus y su hábitat natural. Informe final, 116 pp. Cartagena de Indias, Bolívar.

Ulloa-Delgado G \& D Cavanzo-Ulloa. 2003. Conservación, manejo y uso sostenible de la 'babilla' (Caiman crocodilus fuscus) en la bahía de Cispatá. Etapa I. Caracterización y Diagnóstico de las poblaciones de Caiman crocodilus fuscus y su hábitat natural, 111 pp. Montería, Córdoba.

Velasco A \& J Ayarzagüena. 1995. Situación actual de las poblaciones de Baba (Caiman crocodilus) sometidas a aprovechamiento comercial en los llanos venezolanos. Publicación Asociación Amigos Doñana, Sevilla 5: 19-26. 